\title{
Testosteron macht eigensüchtig
}

\section{Ob wir ideale Teamplayer oder eher eigensinnige Sturköpfe sind, wird offensichtlich vom Testosteron- spiegel bestimmt. Dies ergab eine englische Studie an Frauen.}

- Das berichten Wissenschaftler vom University College London. Die Forscher setzten jeweils Paare aus zwei Frauen vor einen Computermonitor, auf dem nacheinander zwei Bilder auftauchten. In einem der Bilder verbarg sich ein gesuchtes Motiv und die Frauen sollten jeweils entscheiden, in welchem der beiden Bilder es aufgetaucht war. Entschieden sich beide Frauen für dasselbe Bild, ging es weiter zum nächsten Test.

Wann immer beide Kandidatinnen aber ein unterschiedliches Bild wählten, baten die Forscher sie, miteinander zu diskutieren und zu einer einvernehmlichen Entscheidung zu kommen. Die- sen Versuch absolvierten die Frauen im Abstand von etwa einer Woche zweimal. Der Clou dabei: Einmal bekamen sie zuvor eine Dosis Testosteron verabreicht, beim anderen Mal ein Placebo.

Die Auswertung des Tests zeigte, dass die Frauen in der Placebogruppe eher zur Kooperation bereit waren und eine höhere Trefferquote erzielten.

Hatten sie jedoch einen erhöhten Testosteron-Spiegel, verhielten sie sich viel weniger kooperativ und beharrten häufiger auf ihrer eigenen Meinung. Dadurch nahm die Trefferquote des Teams insgesamt ab.

\section{Kommentar}

Das Hormon Testosteron schränkt kooperatives Verhalten ein und lässt Menschen egoistischer handeln. Eine frühere Studie hatte gezeigt, dass Testosteron jedoch auch die Bereitschaft zur Kooperation erhöhtaber nur, wenn sich die Beteiligten dadurch materielle Gewinne oder gesellschaftliches Ansehen erhoffen.

K. MALBERG

- N. D. Wright et al. (Wellcome Trust Centre for Neuroimaging Institute of Neurology, University College London, 12 Queen Square, London WC1N 3BG, UK) Testosterone disrupts human collaboration by increasing egocentric choices. Published online on 11 January 2012 in Proc. Royal. Soc. B

\section{Achtung:}

\section{Hier muss der} Dummy durch eine Anzeige ersetzt werden !! 